C-A/AP/\#154 July 2004

\title{
Uniform Beam Distributions at the Target of the NSRL Beam Transfer Line
}

N. Tsoupas, S. Bellavia, R. Bonati, K. Brown, I-Hung Chiang, C.J. Gardner, D. Gassner, S. Jao, I. Marneris, A. McNerney, D. Phillips, P. Pile, R. Prigl, A. Rusek, L. Snydstrup, BNL

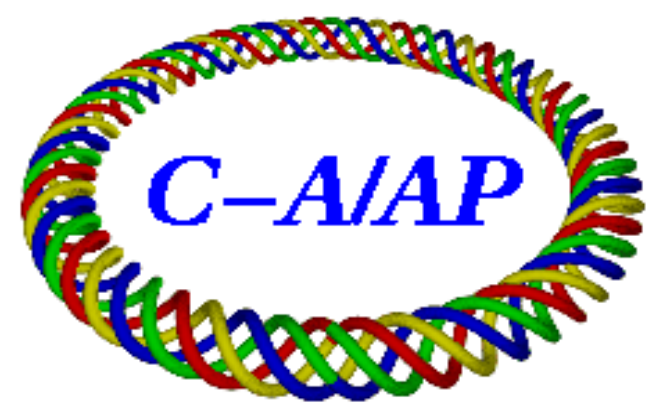

Collider-Accelerator Department Brookhaven National Laboratory Upton, NY 11973 


\title{
Uniform Beam Distributions at the Target of the NSRL Beam Transfer Line
}

\author{
N. Tsoupas, S. Bellavia, R. Bonati, K. Brown, I-Hung Chiang, C.J. Gardner, D. \\ Gassner, S. Jao, Ioannis Marneris, A. McNerney, D. Phillips, P. Pile, R. Prigl, \\ A. Rusek, L. Snydstrup, BNL
}

\begin{abstract}
The NASA SPACE RADIATION LABORATORY (NSRL) has been constructed and started operations at the Brookhaven National Laboratory in 2003. The NSRL facility will be used by NASA to conduct radiation effect studies on materials and biological samples for the space program. The NSRL facility utilizes proton and heavy-ion beams of energies from 50 to $3000 \mathrm{MeV} / \mathrm{n}$ which are accelerated by the AGS Booster synchrotron accelerator. To date, protons, ${ }^{12} \mathrm{C},{ }^{56} \mathrm{Fe},{ }^{48} \mathrm{Ti}$ ion beams of various magnetic rigidities have been transported to the sample location which is located $100 \mathrm{~m}$ downstream from the beginning of the NSRL line. The NSRL beam transport line has been designed to employ octupole magnetic elements[1] which transform the normal (Gaussian) beam distribution at the location of the sample into a beam with rectangular cross section, and uniformly distributed over the rectangle. To obtain the uniform beam distribution on the sample, no beam-collimation is applied at any location along the NSRL beam transport line and the beam focusing at the location of the sample is purely magnetic. In this technical note we present data from the beam distribution at the target when the octupoles are excited, and compare the beam distributions with octupoles turned off, and the effects of beam misalignments on the beam uniformity at the target will be discussed along with the correction methods used. Future developments on obtaining uniform beams will also be discussed.
\end{abstract}

\section{Introduction}

The NSRL facility has been constructed at BNL to be used by NASA and other scientific organizations to conduct irradiation experiments on materials and biological samples. The construction of the NSRL beam line has been completed in March 2003 and the commissioning of the beam transport line has been completed in June 2003.

The ion beams which are available by the facility for the irradiation of the samples are being accelerated by the BNL Booster and transported to the sample by the NSRL beam transport line which is the subject of this paper. Table 1 shows some of the ion beams that have been transported to the sample by the NSRL beam transport line. Unlike most of the beam transport lines which utilize only dipole and quadrupole magnetic elements for beam transport and focusing, the NSRL beam transport line has a unique characteristic that employs octupole magnetic elements to generate uniform irradiation fields at the location of the sample.

Uniform beam irradiation fields, over relatively large areas $20 \times 20 \mathrm{~cm}^{2}$, are often required to irradiate materials or biological samples. In other laboratories such uniform beam distributions are obtained using various methods depending of the ion beams[2]. For protons and other light ions, uniform beams are obtained by increasing the angular divergence of the beam, by letting the beams passing through a layer of material that 
multiple scatters the beam to increases the beam divergence. This technique has the following drawback:

a) Only a small fraction of the beam is being used by the sample with the larger fraction of the beam remaining unused. The reason is that the beam uniformity is achieved by the multiple scattering of the beam in a material to increase the angular divergence of the beam and obtain a large spatial standard deviation of the "normally" distributed beam at the location of the sample.

b) Activation of the material that is used to increase the divergence of the beam through the multiple scattering.

c) The sample is being irradiated not only by the ions of the beam but also with ions produced from the nuclear fragmentation that occurs when the beam passes through the scattering material which is used to generate the uniform distribution.

For heavier ions that have higher rigidity, the multiple scattering technique in not effective therefore the "magnetic rastering" technique is used. In this method the beam is focused into a small spot at the location of the sample and the spot of the beam is sweeping the sample. The beam sweep is achieved by deflecting the beam horizontally and vertically using dipole magnets which are located upstream of the sample. The disadvantages of this technique is:

a) the beam must be constrained to have constant intensity during the irradiation period. This constrain requires sophisticated control over the extracted beam and is not easily obtained.

b) The reliability of the rastering system because of its complexity.

The drawbacks of the techniques, mentioned above, to generate uniform irradiation over the samples are eliminated by using octupole elements to modify the Gaussian beam distribution at the location of the sample to a uniform one. An experimental "proof of principle" of this method appears in Ref.[3]. A detailed description of the theoretical aspect of this method appears in Ref.[1]. In this paper we present results from the commissioning of the NSRL beam transport line which employs octupole elements to generate uniform beam distributions over the sample. The following aspects from the commissioning of the NSRL beam transport line are presented.

a) Description of the NSRL beam transfer line. (magnet layout, beam envelop of the line using first order beam optics)

b) Measured beam parameters at the beginning of the NSRL line and experimental first order beam envelope.

c) Beam distribution at the location of the target with and without the use of octupoles.

d) Effect of the magnets instability, and beam misalignment on the beam uniformity at the sample.

e) The beam coupling due to octupole magnetic elements.

f) Further development of generating uniform beam distributions using duodecapole magnetic elements. 


\section{NSRL Beam Transfer Line (Layout and First Order beam optics)}

\section{Magnetic elements of the NSRL beam transport line}

The layout of the magnetic elements which comprise the NSRL beam transfer line are shown in Fig 1. The magnetic elements with the order that appear in the NSRL line are:

a) D6: A $9.8^{0}$ Left-bend dipole septum magnet which extracts the beam from the AGS booster.

b) Q1,Q2: quadrupole magnets

c) D20: A $20^{0}$ left-bend dipole magnet.

d) Q3, Q4: Quadrupoles

e) O1: Horizontally defocusing Octupole; when excited, modifies the horizontal Gaussian beam distribution at the target into a uniform distribution.

f) Q5: Quadrupole magnet

g) $\quad$ 2: $\quad$ Vertically focusing Octupole. Its function is similar to the octupole O1 upstream but this octupole acts on the vertical beam distribution.

h) Q6,Q7,Q8: Quadrupoles to be used to adjust the shape of the beam at the target.

Beam constraints along the NSRL beam transfer line. Apart from the required constraints (aperture ...,topology of the building) that every beam line should satisfy, additional constraints are imposed on the optics of the NSRL beam transfer line.

These constraints are:

a) The magnetic elements D6,Q1,Q2, and D20 ${ }^{\circ}$ form an achromatic bend. Therefore the beam at the exit of the of the D20 dipole magnet is achromatic, under the condition that the beam at the entrance of the D6 septum magnet is achromatic.

b) The $\left(x, x^{\prime}\right)$ beam particle coordinates at the location of the octupole (O1) should be highly correlated in the horizontal plane[2] to facilitated the effect of the octupole magnetic element which will generate the uniform beam at the target on the horizontal direction. Similar constraint should also apply for the particle_beam coordinates $\left(\mathrm{y}, \mathrm{y}^{\prime}\right)$ at the location of the second Octupole $(\mathrm{O} 2)$.

TABLE 1 Some of the ion species that have been transported at the target location of the NSRL beam transport line.

\begin{tabular}{|c|c|c|c|}
\hline Ion & Energy[MeV/nucleon] & $\begin{array}{c}\text { Charge state } \\
\text { before stripping }\end{array}$ & $\begin{array}{c}\text { Charge state after } \\
\text { stripping }\end{array}$ \\
\hline $\mathrm{p}$ & 730 & +1 & +1 \\
\hline${ }^{12} \mathrm{C}$ & 300 & +5 & +6 \\
\hline${ }^{56} \mathrm{Fe}$ & 1000 & +20 & +26 \\
\hline${ }^{48} \mathrm{Ti}$ & 1000 & +18 & +22 \\
\hline${ }^{28} \mathrm{Si}$ & 600 & +9 & +14 \\
\hline
\end{tabular}




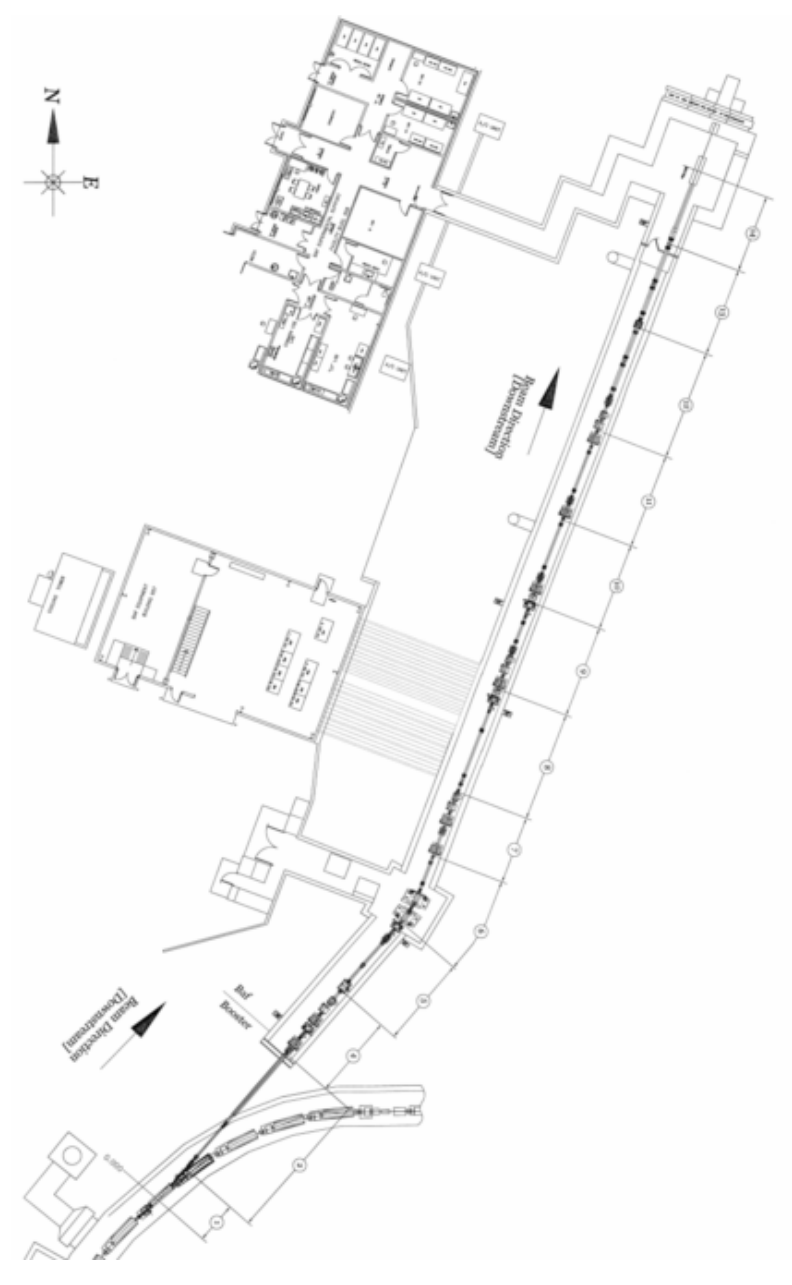

Figure 1. Drawing of the NSRL beam transfer line. The sector at the bottom of the picture is part of the Booster ring.

\section{First order beam Optics}

The first order beam optics that satisfies the constraints mentioned earlier has been worked out using the TRANSPORT or MAD computer codes. In the model of the NSRL line we have assumed that the beam parameters of the extracted beam at the beginning of the NSRL beam transport line are identical to the beam parameters of the circulating beam at the same location. Table 2 shows the theoretical horizontal and vertical beam parameters at the beginning of the NSRL beam transport line. These parameters were used during the design of the NSRL line. 
TABLE 2. The assumed (Theoretical) and measured beam parameters at the beginning of the NSRL transport line. The "nm" indicates "not measured"

\begin{tabular}{|c|c|c|c|c|c|c|c|c|}
\hline & $\alpha_{\mathrm{x}}$ & $\beta_{\mathrm{x}}[\mathrm{m}]$ & $\eta_{\mathrm{x}}[\mathrm{m}]$ & $\eta_{\mathrm{x}}^{\prime}$ & $\alpha_{\mathrm{y}}$ & $\beta_{\mathrm{y}}[\mathrm{m}]$ & $\eta_{\mathrm{y}}[\mathrm{m}]$ & $\eta_{\mathrm{y}}^{\prime}$ \\
\hline Theor. & 1.87 & 10.0 & 0.0 & 0.0 & 0.637 & 4.39 & 0.0 & 0.0 \\
\hline Exper & $\begin{array}{c}0.3 \\
( \pm 0.2)\end{array}$ & $\begin{array}{c}10.6 \\
( \pm 3.0)\end{array}$ & $\mathrm{nm}$ & $\mathrm{nm}$ & $\begin{array}{c}-0.51 \\
( \pm 0.2)\end{array}$ & $\begin{array}{c}7.4 \\
( \pm 1.2)\end{array}$ & $\mathrm{nm}$ & $\mathrm{nm}$ \\
\hline
\end{tabular}

We have also assumed that the beam at the beginning of the NSRL line is normally distributed in all of the coordinates. In fact in order to ascertain that the slow extracted beam acquires a normal distribution at the beginning of the NSRL line the beam is usually passing through a thin foil which is inserted at the entrance of the D6 septum magnet. A set of thin foils and thin wires have been mounted on a wheel which is placed at the entrance of the D6 septum. A particular foil/wire is inserted in the beam by rotating the wheel at the proper orientation. The effect of the foil on the beam is twofold, first the ions of the beam are stripped from any of the electrons before it enters the NSRL line, thus reducing the rigidity of the extracted beam, and second the beam acquires a Gaussian distribution through the multiple scattering of the beam in the foil. We usually insert in the beam the $\mathrm{Cu}$ foils of thickness $0.5 \mathrm{~mm}$ or $0.05 \mathrm{~mm}$. Table 1 shows the charge states, before and after stripping, of some of the ions transported by the NSRL line. In figure 2 plotted are the horizontal and vertical beta functions $\beta_{\mathrm{x}}$ and $\beta_{\mathrm{y}}$ along the NSRL beam transfer line for a particular beam focusing. This beam focusing will generate a beam with rectangular cross section and uniform distribution within the rectangle, when the octupole magnetic elements are excited. The beam parameters at the location of the octupoles are constrained, to provide the required first order beam focusing for the octupoles to generate the rectangular and uniform beam distribution at the location of the target which is located $100 \mathrm{~m}$ downstream from the beginning of the NSRL line.

Figure 2 shows also the location of the magnetic elements (blue boxes) of the NSRL beam transfer line. The same magnetic elements are also shown, but labeled, in figure 3 . Figure 3 shows half of the horizontal and vertical beam profiles plotted along the NSRL beam transfer line. The beam profiles shown in figure 3 are generated from the beta functions shown in figure 2. and the beam emittance which is assumed to be $10 \pi[\mathrm{mm} . \mathrm{mrad}](95 \%$ normalized) for either the horizontal and vertical planes.

Instrumentation packages have been installed at specified location along the NSRL beam transfer line. These packages can be inserted in the beam path, or retracted from, to monitor the beam intensity and the beam profiles along the NSRL line for beam diagnostic purposes. The beam intensity is measured with the Ionization Chambers (IC) 
and the beam profiles are measured, with the Segmented Wire Ionization Chambers (SWIC). Both devices IC and SWIC are part of the instrumentation packages. The two dimentional beam profiles can be measured with the Visual_Flags (VF). Each of the flags is located $0.75 \mathrm{~m}$ upstream from each instrumentation package. A model of the NSRL line has been generated to be used as an input in the TRANSPORT[4], or MAD[5] or SPRAY[6] computer code. The third order calculations have been performed with the TRANSPORT or SPRAY computer codes. A MAD input model of the NSRL line has been included in APPENDIX 1.

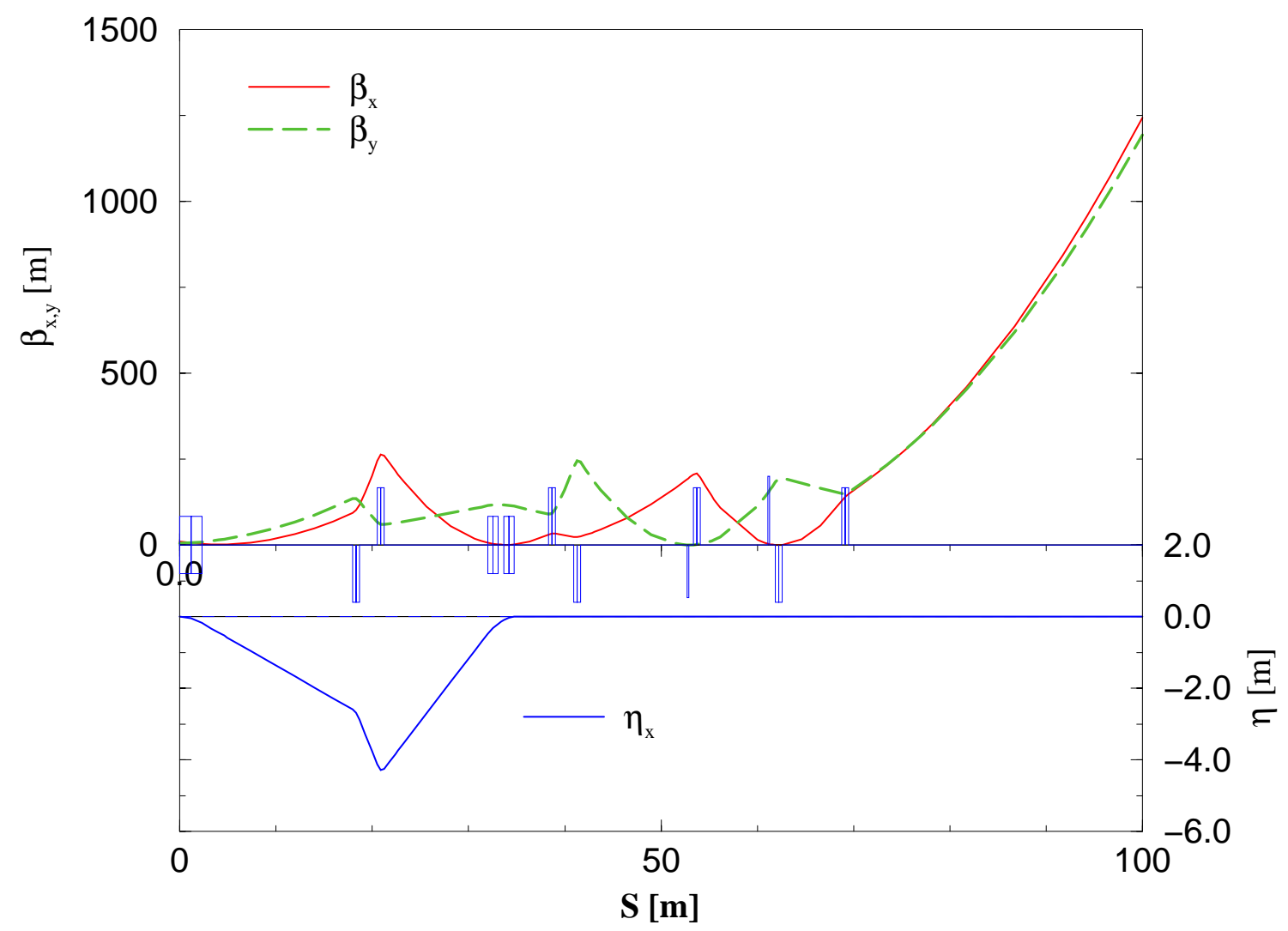

Figure 2. The horizontal and vertical beta functions along the NSRL transfer line. The magnetic elements shown as rectangular boxes are labeled in figure 3 .

The strength of the dipole and quadrupole magnetic elements which are used by the MAD input model to yield the beam parameters $\left(\beta_{\mathrm{x}}, \beta_{\mathrm{y}}\right.$ shown in fig. 2$)$ and the beam profiles (shown in fig. 3 ) along the NSRL beam transfer line, are shown in TABLE 3.

TABLE 3: The strength of the magnetic elements for the first order beam optics which is used to generated uniform beam at the target when the octupoles turn ON.

\begin{tabular}{|l|l|l|l|l|l|l|l|l|}
\hline D6 & Q1 & Q2 & D20 & Q3 & Q4 & Q5 & Q6 & Q7 \\
\hline$[\mathrm{mrad}]$ & {$\left[\mathrm{m}^{-1}\right]$} & {$\left[\mathrm{m}^{-1}\right]$} & {$[\mathrm{mrad}]$} & {$\left[\mathrm{m}^{-1}\right]$} & {$\left[\mathrm{m}^{-1}\right]$} & {$\left[\mathrm{m}^{-1}\right]$} & {$\left[\mathrm{m}^{-1}\right]$} & {$\left[\mathrm{m}^{-1}\right]$} \\
\hline 155.3 & 0.1891 & 0.2393 & 349.06 & 0.3007 & 0.2537 & 0.1755 & 0.1309 & 0.0819 \\
\hline
\end{tabular}


The beam profiles along the NSRL beam transfer line as measured by the SWIC's, have been used to calculate the beam parameters $\left(\beta_{\mathrm{x}, \mathrm{y}}, \alpha_{\mathrm{x}, \mathrm{y}}\right)$ and emittance $\left(\varepsilon_{\mathrm{x}, \mathrm{y}}\right)$ at the beginning of the NSRL beam transfer line. From the measured beam profiles, the experimental beam parameters at the beginning of the NSRL line can calculated. The experimentally measured beam parameters of a $1.0 \mathrm{GeV} /$ nucleon Ti beam after passing through a 0.002 " $\mathrm{Cu}$ foil are shown in the third row of Table 2 . The measured " $95 \%$ Normalized" beam emittance of the Ti beam was $\varepsilon_{\mathrm{y}}=4.3 \pm 1 \pi[\mathrm{mm} . \mathrm{mrad}]$ and $\varepsilon_{\mathrm{y}}=2.6 \pm 0.5$ $\pi[\mathrm{mm} . \mathrm{mrad}]$ for the horizontal and vertical plane respectively.

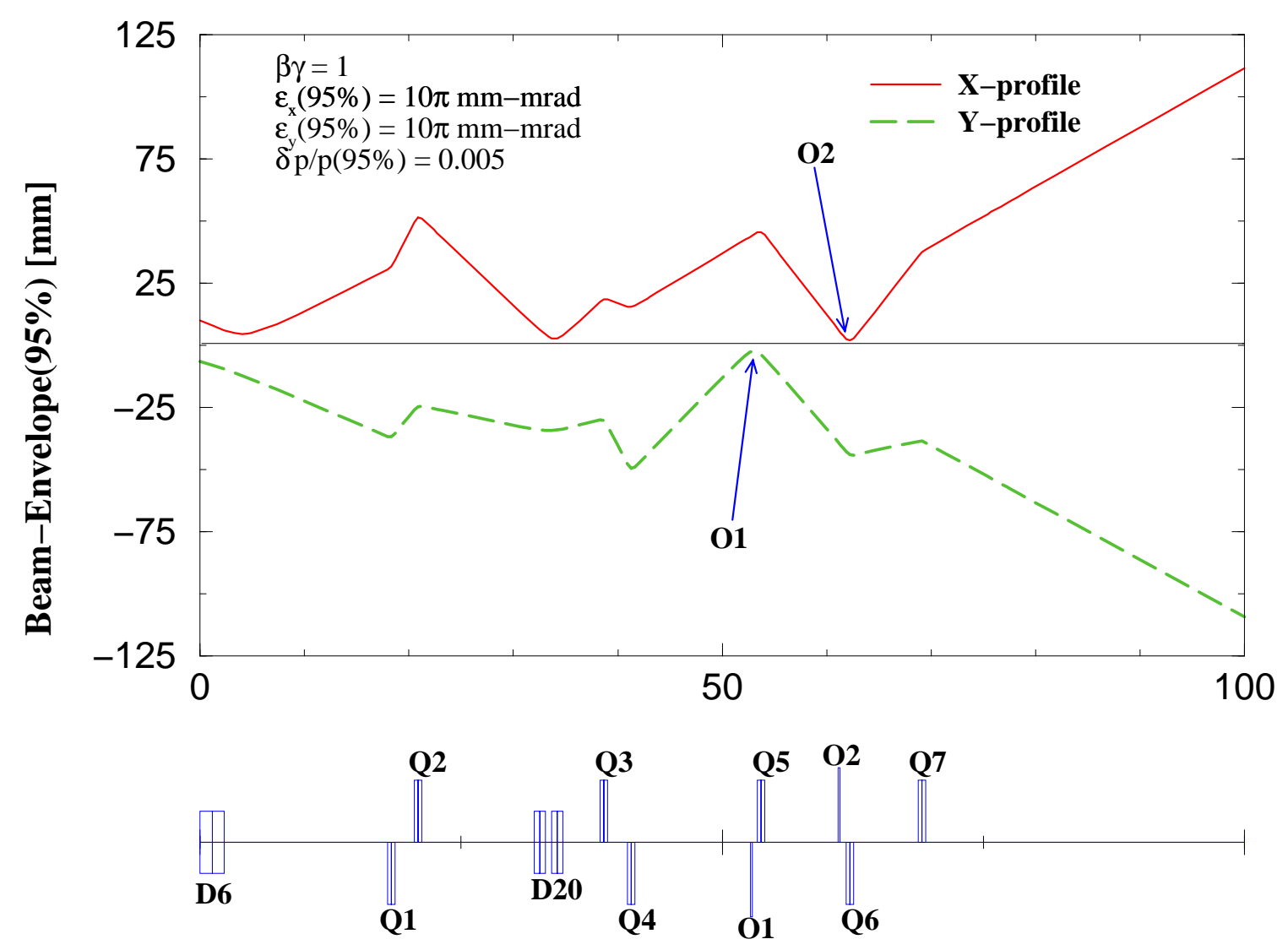

Figure 2. Half of the horizontal (red solid line) and half of the vertical (bottom dashed line) beam profile of the beam transported in the NSRL transport line. The profiles correspond to a $95 \%$ normalized beam emittance of $10 \pi[\mathrm{mm} . \mathrm{mrad}]$ and are generated using first order beam optics (octupoles are not excited).

\section{Third order beam Optics}

The first order beam optics, that satisfies the beam constraints mentioned earlier, provides the beam conditions for the octupoles (when excited) to transform the "normally" distributed beam on the target into a beam with rectangular cross section uniformly distributed over the rectangle.

The theory that describes the action of the octupoles on the beam distribution, which is assumed to be "normal" in all of its coordinates, is described in details in ref. [1]. In this 
paper we only present experimental results from the measured beam distributions at the target, with the octupoles turned on and off. Figure 4 shows the horizontal and vertical projections of the beam distribution at the target when the octupoles of the NSRL beam transfer line are off. These beam distributions which are measured with the SWIC's are Gaussian. Figure 5 shows the corresponding projections of the beam distributions along the $\mathrm{x}$ and $\mathrm{y}$ directions, when the octupoles are on. Comparing the projections of the beam distributions of figures 4 and 5 we can conclude that the octupoles transform the Gaussian beam distribution into a uniform distribution which falls off quickly to zero outside the radiation region. Apart from the increase of the beam intensity at the edges of the distribution the beam intensity is rather uniform within the distribution of the beam. The increase of the beam intensity at the edges of the distribution is explained in a section below.

A better way to visualize the extend of the beam uniformity within the irradiated rectangle which is normal to the beam direction, is shown in figure 6 . This figure has been obtained by a visual flag which is located close to the target location, and shows the beam intensity as a function of the transverse $(\mathrm{x}, \mathrm{y})$ beam coordinates. The figure shows that the beam intensity at the target is confined within a rectangle, it is uniform within the rectangle and exhibits an increase of intensity at the perimeter of the rectangle. The projection of this two dimensional distribution along, either the $\mathrm{x}$ or $\mathrm{y}$ axis generates the one dimensional distributions shown in figure 5.

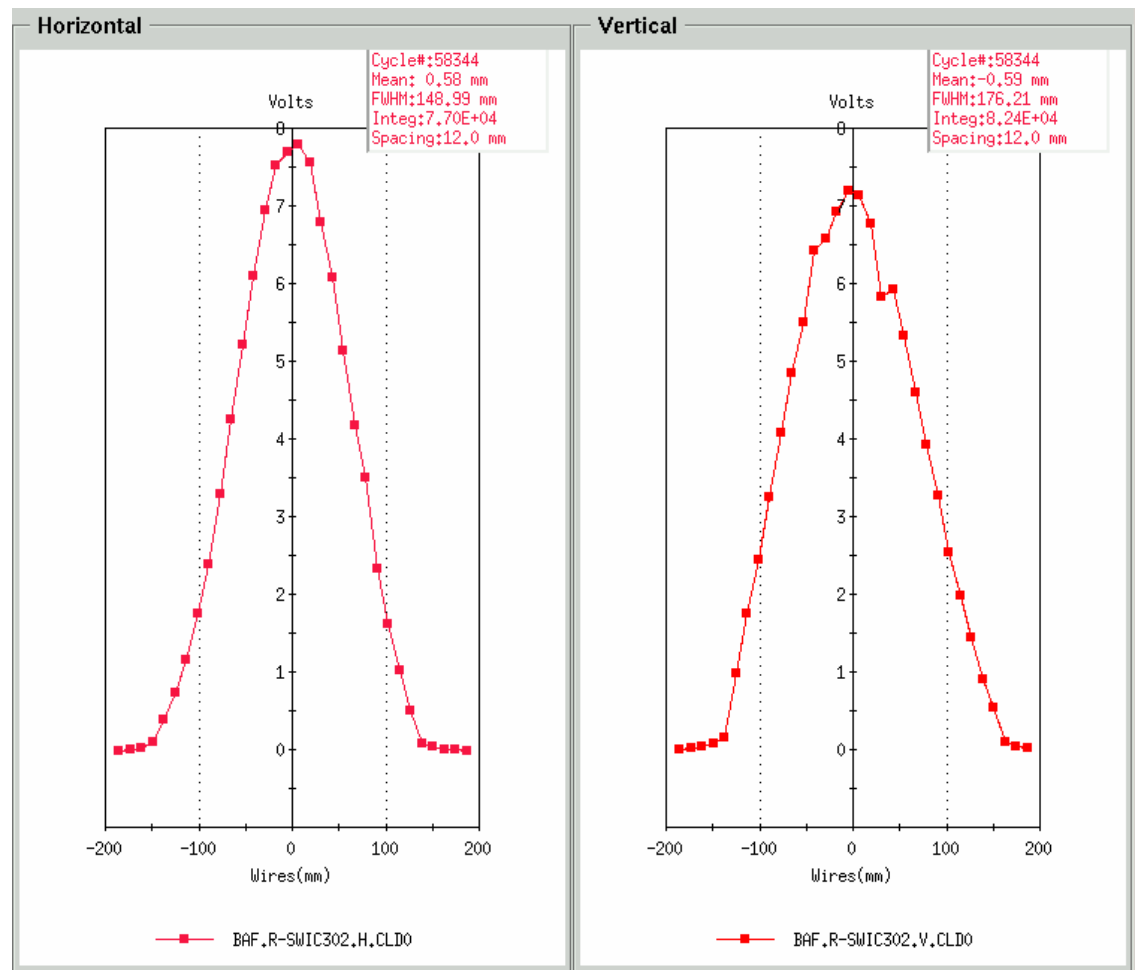

Figure 4. The horizontal and vertical projections of the beam distribution at the NSRL target location, when the octupoles shown in figures 2 or 3 are off. 


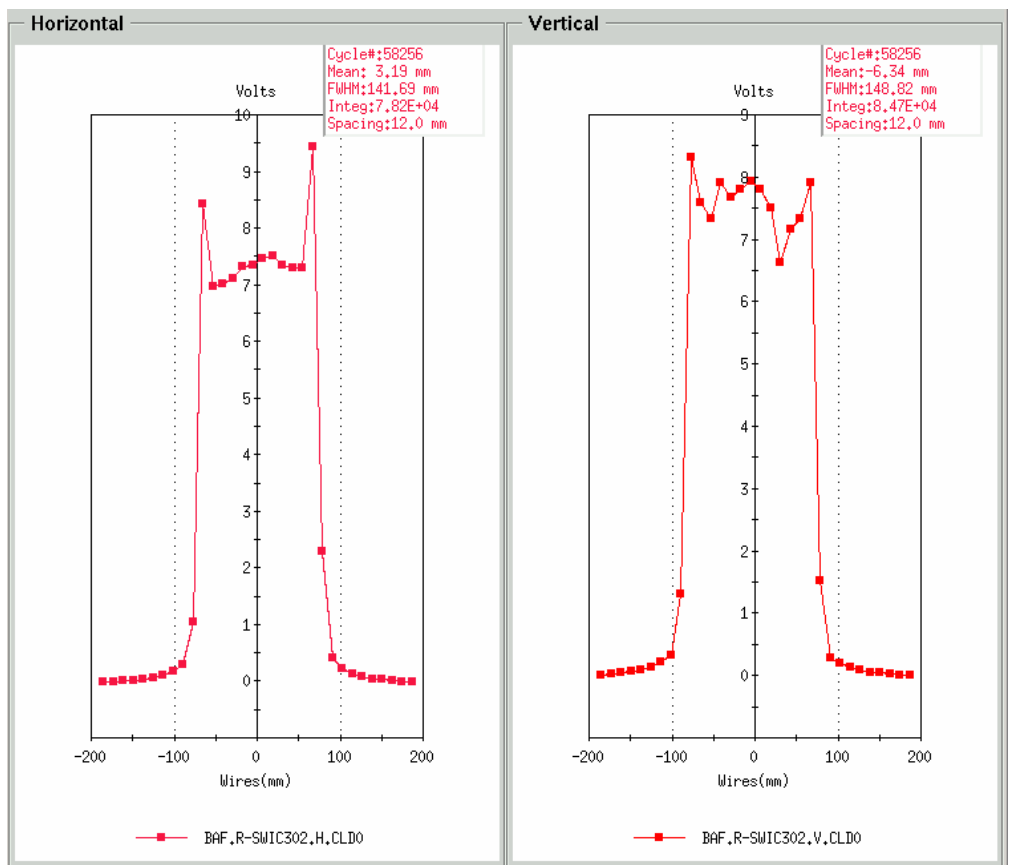

Figure 5. The horizontal and vertical projections of the beam distribution at the NSRL target location, when the octupoles O1, O2 shown in figures 2 or 3 are on. The sudden dip of the beam distribution which appears in the vertical wire located at $\sim 45 \mathrm{~cm}$ is due to the different electronic gain of this particular wire.

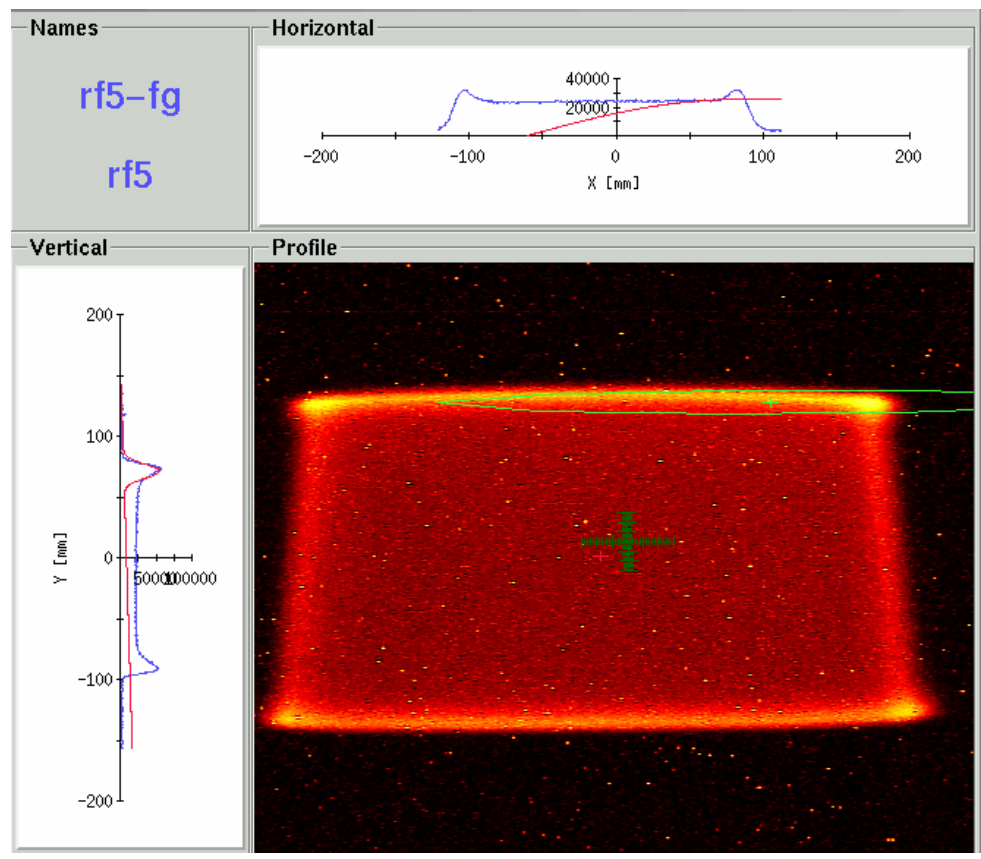

Figure 6. A two dimensional beam distribution obtained on a visual flag when the octupoles are on. This beam distribution corresponds to the projected beam distributions shown in figure 5. 


\section{Effect of beam misalignments on the uniformity of the beam profile at the target.}

During a regular setup of the NSRL beam transport line for uniform beam (Octupoles are on) irradiation of the target, it is possible that the beam distribution on the target is not uniform and its projection on the horizontal or vertical plane or both, may look like the profiles shown in figure 7. Such a distribution as shown in figure 7 is an indication that the beam centerline is not lined up with the magnetic axis of the octupole which responsible to affect the horizontal distribution of the beam at the target. The nonuniform distribution shown in figure 7 can become uniform by correcting the beam misalignment through the excitation of a horizontal or vertical corrector which will place the center of the beam on the octupole.

Computer simulations explain the horizontal beam profile that appears in figure 7 . Indeed figure 8 shows the computer simulation that generated by:

a) a beam profile at the target normally distributed

b) a uniform beam profile when the octupole is $\mathrm{ON}$, and

c) an asymmetric beam profiles by displacing the beam horizontally by $5 \mathrm{~mm}$ from the magnetic axis of the octupole $\mathrm{O} 1$ which is $\mathrm{ON}$.

The beam profiles shown in figure 8 are the projections along the horizontal axis of each of the three phase spaces $\left(x, x^{\prime}\right)$ that appear in figure 9 . Figure 9 shows the Horizontal phase space $\left(x, x^{\prime}\right)$ of the beam distribution at the target for the three different cases $\left.a\right), b$ ) , c) mentioned earlier. When the octupole is OFF the distribution of the phase space $\left(\mathrm{x}, \mathrm{x}^{\prime}\right)$ forms an ellipse as expected. However when the octopole is $\mathrm{ON}$, its action on the beam is to transform the elliptical beam distribution of the phase space $\left(\mathrm{x}, \mathrm{x}^{\prime}\right)$ (black dots) into an "S" shape phase space $\left(\mathrm{x}, \mathrm{x}^{\prime}\right)$ distribution (read dots). When the beam is displaced in front of the octupole, the action of the octupole on the beam is asymmetric, as a result the " $\mathrm{S}$ " shape phase space $\left(\mathrm{x}, \mathrm{x}^{\prime}\right)$ distribution on the target becomes asymmetric (green dots). The projection of each of the three phase space $\left(\mathrm{x}, \mathrm{x}^{\prime}\right)$ distributions on the horizontal axis, generates each of the beam profiles appearing in figure 7 . 


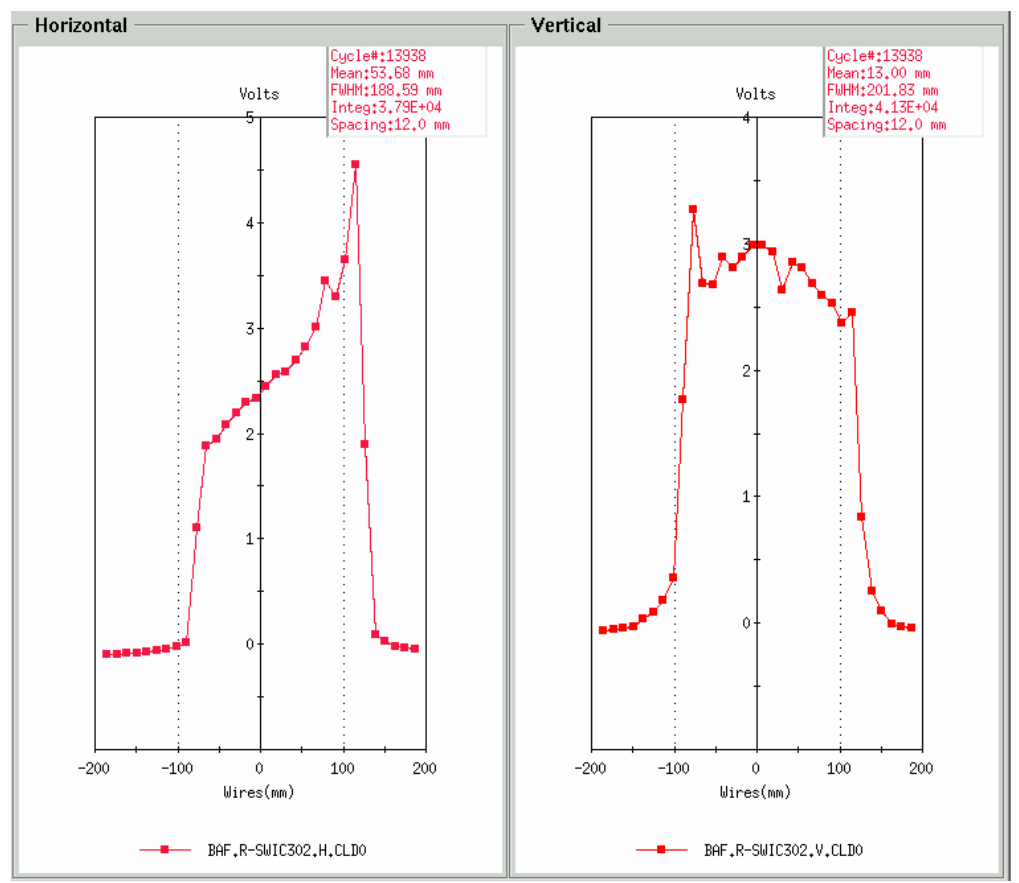

Figure 7. Horizontal and vertical projections of the beam distribution at the target location with octupoles ON. This experimental beam distribution has been generated by displacing horizontally the center of the beam at the location of the octupole O1, away from the axis of the octupole.

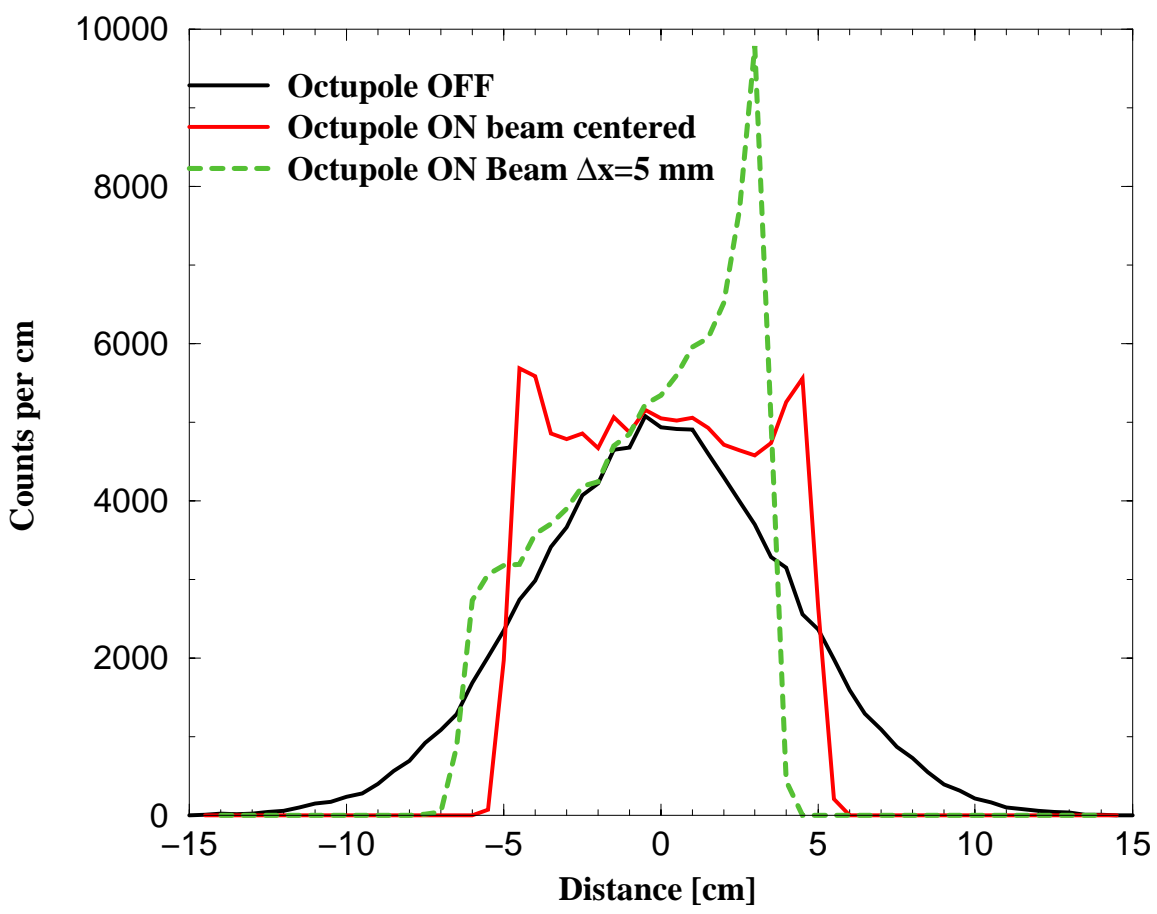

Figure 8. Computer simulations of Horizontal beam profiles on the target for three different cases (see text). The parallel beam displacement from the center of the octupole generates the "slanted" beam distribution on the target. 


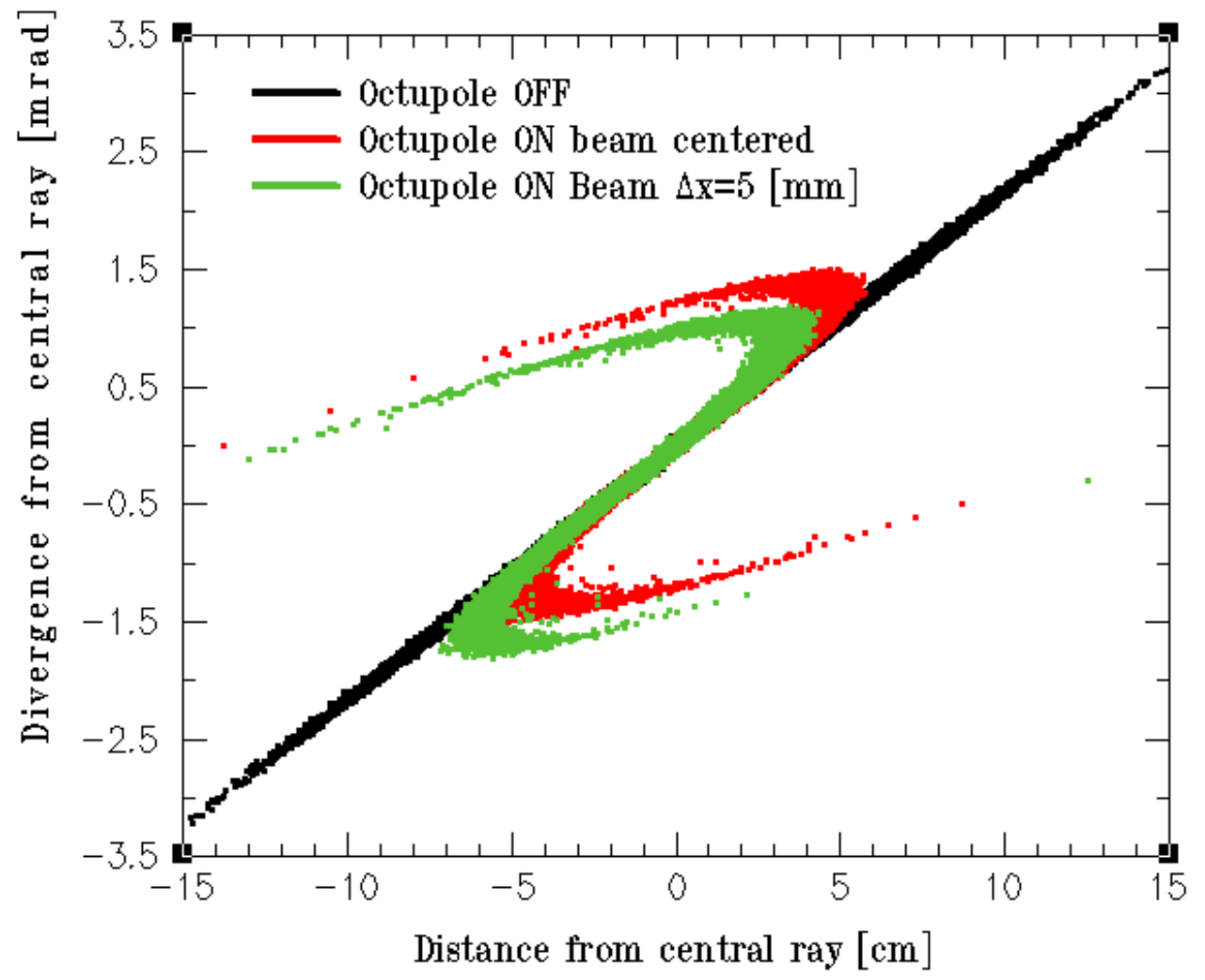

Figure 9. Horizontal phase space $\left(\mathrm{x}, \mathrm{x}^{\prime}\right)$ of the beam distribution at the target. Note that the octupole transforms the elliptical beam distribution (black dots) into an " $\mathrm{S}$ " shape $(\mathrm{x}, \mathrm{x}$ ') distribution. The projection of each of the three distributions generates each of the beam profiles appearing in figure 8.

\section{Beam Coupling due to the Octupole elements.}

The excitation of the octupole magnetic elements is a cause for beam coupling. Computer simulations show that the beam coupling is not significant due to the beam constraints, imposed by the first order beam optics at the location of the octupoles. The minimal beam coupling can be also demonstrated by the measurements of the beam profiles at the location of the target with one of the octupoles turned on and the other off.

Figure 10 shows the horizontal and vertical beam profiles at the location of the target with the octupole that affects the horizontal beam profile turned on and the octupole that affects the vertical beam profile turned off. The Gaussian shape of the vertical beam profile shown in figure 10 indicated that the octupole that affects the horizontal beam profile does not affect the vertical beam profile. Figure 11 demonstrates that, when the octupole that affect the vertical beam profile is turn on and the octupole that affects the vertical beam profile is off, the "vertical" octupole has a negligible effect on the horizontal beam profile of the beam which maintains its Gaussian shape. 
In few of the two dimensional beam distributions obtained by the visual flag located at the target shows that the cross section of the beam is not always rectangular (see fig. 6). This indicates that the beam distribution at the beginning of the NSRL beam transport line is not purely Gaussian.

\section{Further developments}

Preliminary computer simulations show that by replacing of the octupole magnetic elements with duodecapoles magnetic elements, one, can generate also uniform beam at the target with the added benefit of reduction of the beam intensity at the perimeter of the rectangle. The above statement is based on a simple models that generate the magnetic fields of either octupole or duodecapole. As part of a study is to compute the magnetic fields of the octupole and duodecapole magnetic elements, using a 3D computer code for electromagnetics, and subsequently to use the computed field to study and compare the effect of the octupoles/duodecapoles on the beam distribution at the target.

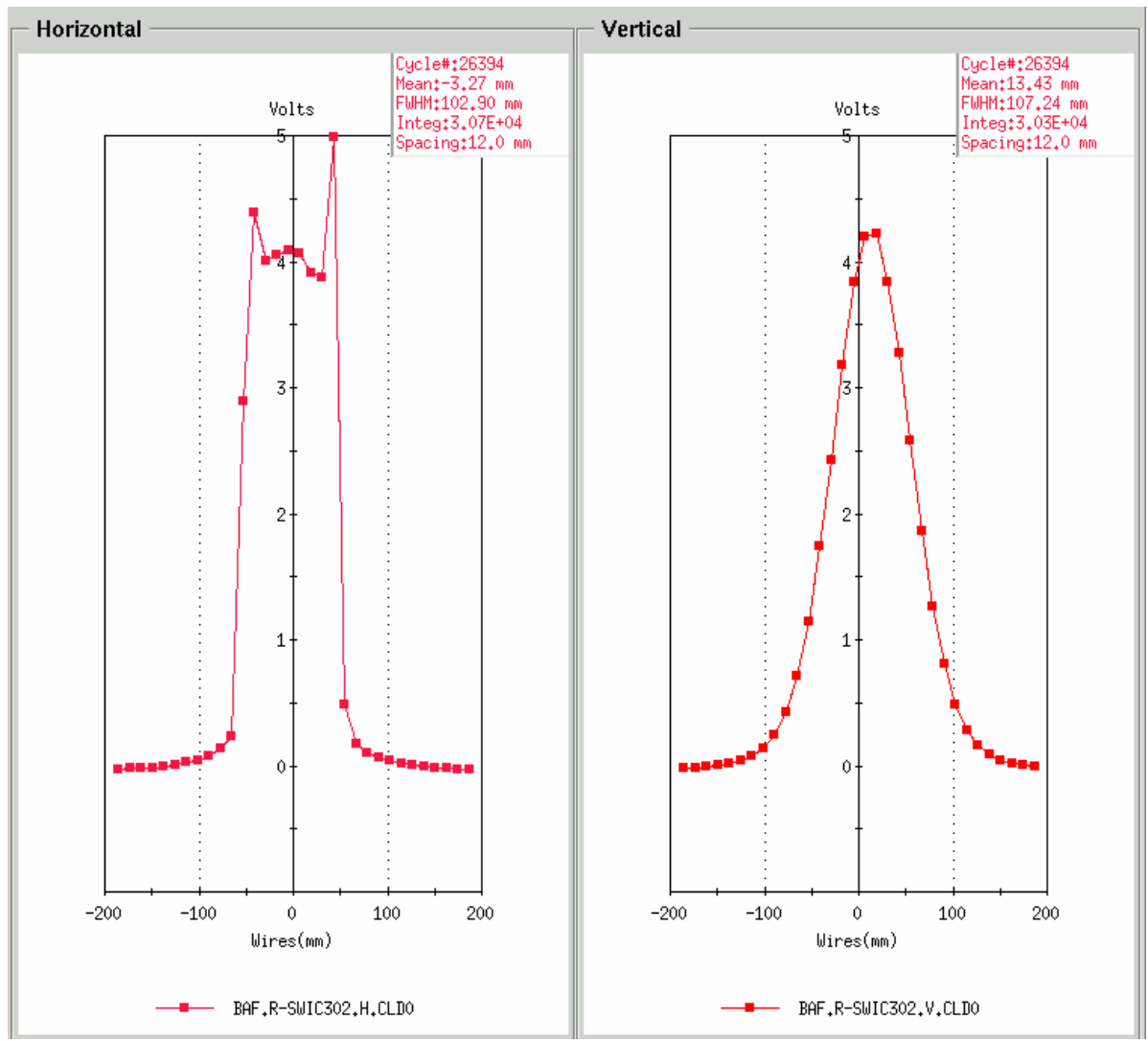

Figure. 10. Horizontal and Vertical beam profiles with the "Horizontal" Octupole ON only. 


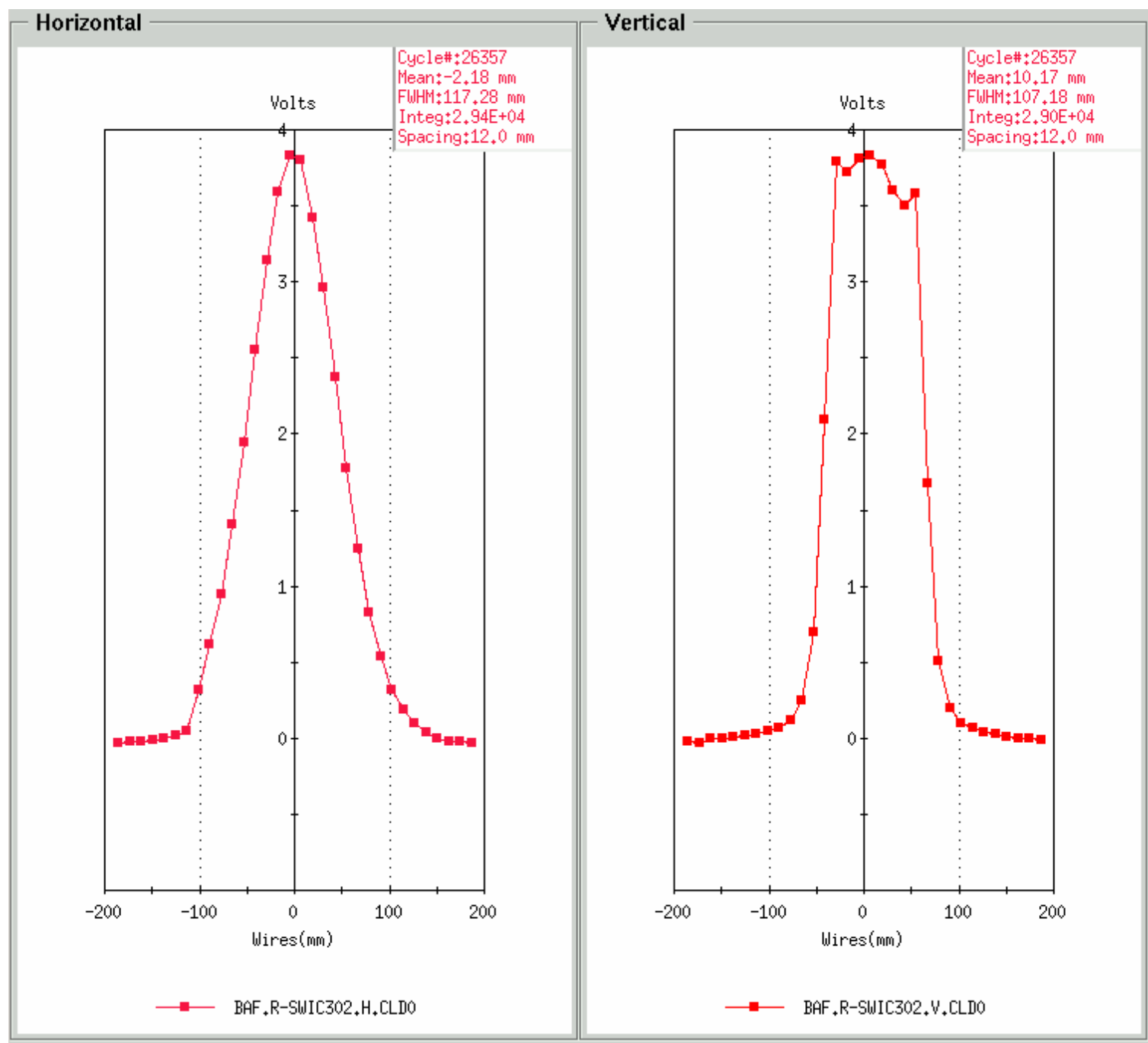

Figure. 11. Horizontal and Vertical beam profiles with the "Vertical" Octupole ON only.

\section{References}

[1] "Uniform Particle Beam Distribution Produced by Octupole Focusing"

N. Tsoupas et. al. Nuclear Science and Engineering: 126, $71-79$ (1997)

[2] "Review of Ion Beam Therapy: Present and Future" J. Alonso LBNL EPAC 2000

Vienna Austria

[3] "Uniform Beam Distributions Using Octupoles" N. Tsoupas et. al. PAC 1991 San

Francisco CA, May 6-9 1991 p.1695

[4] D. C. Carey "Third Order Transport" SLAC-R-95-462

[5] The MAD Program CERN/SL/90-13 (AP)

[6] N. Tsoupas BNL Private Comunication. 


\section{APPENDIX 1}

MAD_model of the NSRL Beam Transport Line

TITLE, " BOOSTER APPLICATION FACILITY BEAM LINE"!

!

KQ1:=-0.266008 !

$\mathrm{KQ} 2:=0.336559 \quad$ !

!

KQ3: $=0.422957 \quad$ !

KQ4:=-0.356860 !

!

KQ5: $=0.246916 !$

KQ6: $=-0.184214$ !

!

KQ7: $=0.115285$ !

KQ8: $=0.000000$ !

!

!---------------------------

!

! ( BAF Line )

!

!LL00: DRIFT, L=1.54598 ! Distance from intersection of (Q6-Q7)and(Q1-Q2) to end of septum

LL00: DRIFT, $\mathrm{L}=0.0$ !

SD1A: SBEND, $\mathrm{L}=1.150$, ANGLE $=-0.0774995, \mathrm{E} 1=-0.012, \mathrm{E} 2=0$ !

SD1B: SBEND, L=1.150, ANGLE $=-0.0774995, \mathrm{E} 1=0.0, \quad \mathrm{E} 2=0$ !

!

LL0A: DRIFT, $\mathrm{L}=0.957732$

LL0B: DRIFT, $\mathrm{L}=0.750824$

LLOC: DRIFT, $\mathrm{L}=0.582574$

!

VK1: VKICKER, $\mathrm{L}=0.0001, \mathrm{KICK}=0.0$ ! $(\mathrm{RP} 11)$

LDK1: DRIFT, $\mathrm{L}=0.337515$ !

HK1: HKICKER, L=0.0001, KICK=0.0 ! (RD13)

!

LS1: DRIFT, L $=2.39461 \quad$ !

LS2: DRIFT, L=2.0 ! (Cleanup Slits)

!

COLL1: RCOLLIMATOR, L=0.00001, XSIZE $=0.10$, YSIZE $=0.10$ ! !

LL0F: DRIFT, L=2.62043 !

LL0G: DRIFT, $\mathrm{L}=2.0$ !

LLOH: DRIFT, $\mathrm{L}=2.0$ ! 
LLOI: DRIFT, $\mathrm{L}=2.0 \quad$ !

!

Q1A: QUADRUPOLE, L $=0.3555, \mathrm{~K} 1=\mathrm{KQ} 1$ !

Q1B: QUADRUPOLE, $\mathrm{L}=0.3555, \mathrm{~K} 1=\mathrm{KQ} 1 \quad$ ! !

LL1A: DRIFT, $\mathrm{L}=0.61156$ !

FL1: MARKER ! (FLAG RF63 )

LL1B: DRIFT, $\mathrm{L}=0.70604$ !

FWS1: MARKER ! (SWIG RW63 SCINT RSC63 )

LL1C： DRIFT, L $=0.5554$ !

!

Q2A: QUADRUPOLE, $\mathrm{L}=0.3555, \mathrm{~K} 1=\mathrm{KQ} 2, \mathrm{TYPE}=\mathrm{QDS}$ !

Q2B: QUADRUPOLE, $\mathrm{L}=0.3555, \mathrm{~K} 1=\mathrm{KQ} 2$, TYPE $=\mathrm{QDS}$ ! !

LL2A：DRIFT, L $=0.5233$ !

!

HK2: HKICKER, L=0.63, KICK $=0.0$ ! $(\mathrm{RD} 70)$

DSK2: DRIFT, $\mathrm{L}=0.2844 \quad$ !

VK2: VKICKER, $\mathrm{L}=0.63, \mathrm{KICK}=0.0$ ! (RP73)

!

LL2B1: DRIFT, $\mathrm{L}=2.34246$ !

LL2B2: DRIFT, $\mathrm{L}=2.5$ !

LL2B3: DRIFT, $\mathrm{L}=2.5 \quad$ !

!

FL2: MARKER ! (FLAG RF92)

LL2C: DRIFT, $\mathrm{L}=0.70612$ !

SWI2: MARKER ! (SWIG RW92 IONCH RI92)

LL2D：DRIFT, $\mathrm{L}=0.64988 ＼mathrm{~ ！ ~} \mathrm{~L}=0.65008$

!

SD2A1: SBEND, $\mathrm{L}=0.53222$, ANGLE $=-0.08726674, \mathrm{E} 1=-0.08726656, \mathrm{E} 2=-0.0$, $\mathrm{HGAP}=0.0762$ !

SD2A2: SBEND, $\mathrm{L}=0.53222$, ANGLE $=-0.08726674, \mathrm{E} 1=-0.0$, $\mathrm{E} 2=-0.08726656$, $\mathrm{HGAP}=0.0762 \quad ! \mathrm{L}=1.067$

!

LLMA： DRIFT, L $=0.30136$ !

LLMB: DRIFT, $\mathrm{L}=0.30136$ !

!

SD2B1: SBEND, L $=0.53222$, ANGLE $=-0.08726674, \mathrm{E} 1=-0.08726656, \mathrm{E} 2=-0.0$, $\mathrm{HGAP}=0.0762 \quad ! \mathrm{L} / 2=0.5335$

SD2B2: SBEND, $\mathrm{L}=0.53222$, ANGLE $=-0.08726674, \mathrm{E} 1=-0.0$, $\mathrm{E} 2=-0.08726656$, $\mathrm{HGAP}=0.0762 \quad ! \mathrm{L}=1.067$

!

FR16: MARKER ! FIT ETAX $=0.0$ ETAX ${ }^{\prime}=0.0$

!

LD2A: DRIFT, $\mathrm{L}=1.59226$ ! $\mathrm{L}=1.59236$

LD2B: DRIFT, $\mathrm{L}=2.0 \quad$ ! 
!

Q3A: QUADRUPOLE, $\mathrm{L}=0.3555, \mathrm{~K} 1=\mathrm{KQ} 3$ !

Q3B: QUADRUPOLE, $\mathrm{L}=0.3555, \mathrm{~K} 1=\mathrm{KQ} 3$ !

!

LL3A: DRIFT, $\mathrm{L}=0.9365$ !

LL3B: DRIFT, $\mathrm{L}=0.9365$ !

!

Q4A: QUADRUPOLE, $\mathrm{L}=0.3555, \mathrm{~K} 1=\mathrm{KQ} 4$ !

Q4B: QUADRUPOLE, $\mathrm{L}=0.3555, \mathrm{~K} 1=\mathrm{KQ} 4$ ! !

LL4A：DRIFT, L=0.5487 !

!

HK3: HKICKER, $\mathrm{L}=0.63, \mathrm{KICK}=0.0$ ! $(\mathrm{RD} 70)$

DSK3: DRIFT, $\mathrm{L}=0.2844$ !

VK3: VKICKER, L=0.63, KICK=0.0! (RP73)

!

L4B1: DRIFT, $\mathrm{L}=2.7665$ !

L4B2: DRIFT, $\mathrm{L}=2.5$ !

L4B3: DRIFT, $\mathrm{L}=2.5$ !

!

FL3: MARKER !(FLAG RF158)

SD3: DRIFT, $\mathrm{L}=0.70612$ !

SWI3: MARKER ! (SWIG RW158 IONCH RI158)

LL4C: DRIFT, $\mathrm{L}=0.43678$ !

!

O1A: DRIFT, $\mathrm{L}=0.2$ ! ( OCTUPOLE \#1A)

O1B: DRIFT, L=0.2! ( OCTUPOLE \#1B)

!

LO1A: DRIFT, $\mathrm{L}=0.3275$ !

!

Q5A: QUAD, L=0.3555, K1=KQ5 ! (Quad \#5 )

Q5B: QUAD, L=0.3555, K1=KQ5 ! (Quad \#5 )

!

LL5A：DRIFT, L=0.5487 !

!

HK4: HKICKER, L=0.63, $\mathrm{KICK}=0.0$ ! $(\mathrm{RD} 178)$

DSK4: DRIFT, $\mathrm{L}=0.2844$

VK4: VKICKER, $\mathrm{L}=0.63, \mathrm{KICK}=0.0$ ! (RP181)

!

LL5B: DRIFT, $\mathrm{L}=3.8555$ !

FL4: MARKER ! (FLAG RF188)

SD4: DRIFT, $\mathrm{L}=0.70612$ !

SWS4: MARKER ! (SWIG RW188 IONCH RI188)

LL5C: DRIFT, $\mathrm{L}=0.43678$ ! 


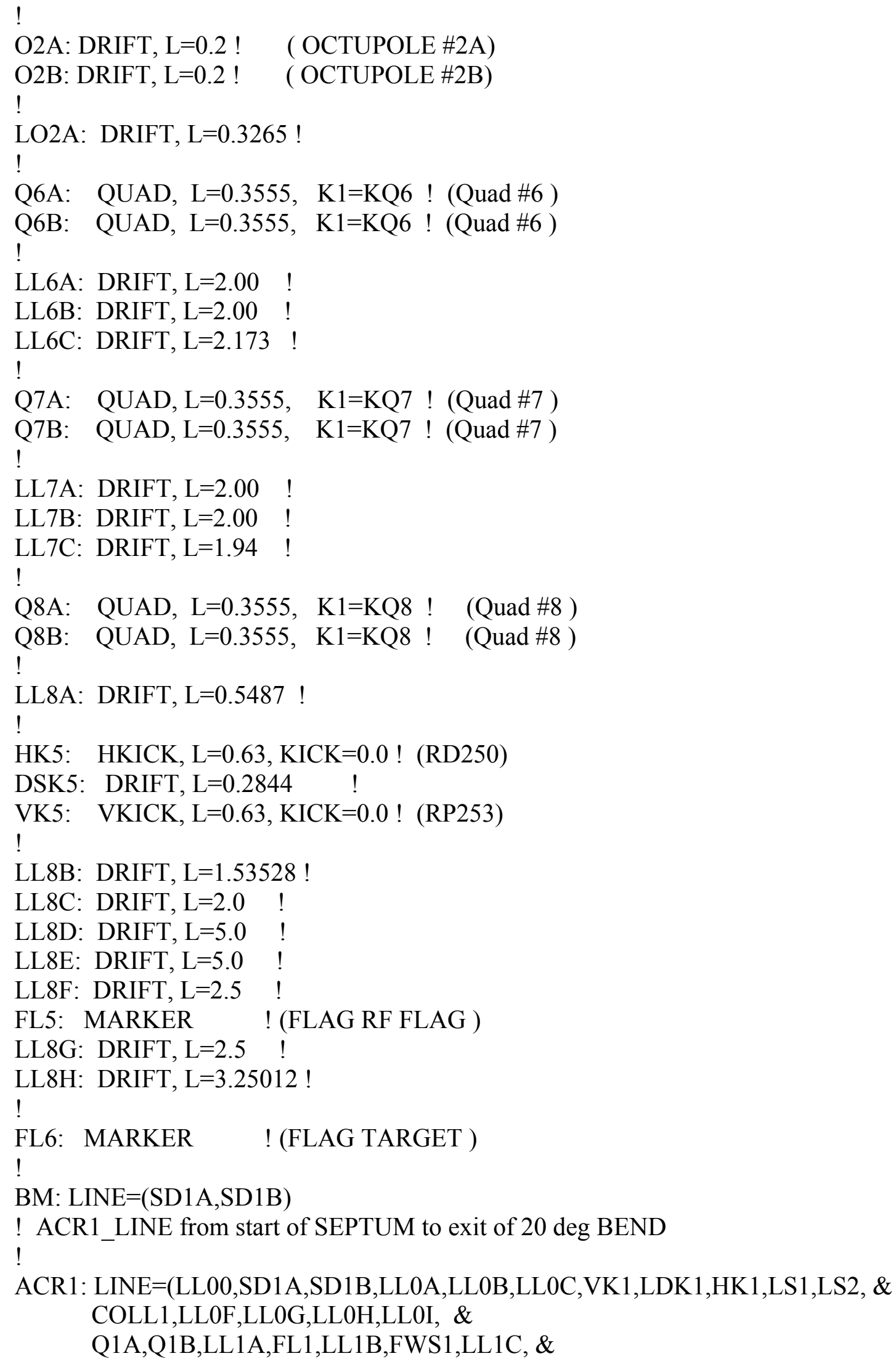
COLL1,LLOF,LL0G,LLOH,LLOI, \& Q1A,Q1B,LL1A,FL1,LL1B,FWS1,LL1C, \& 


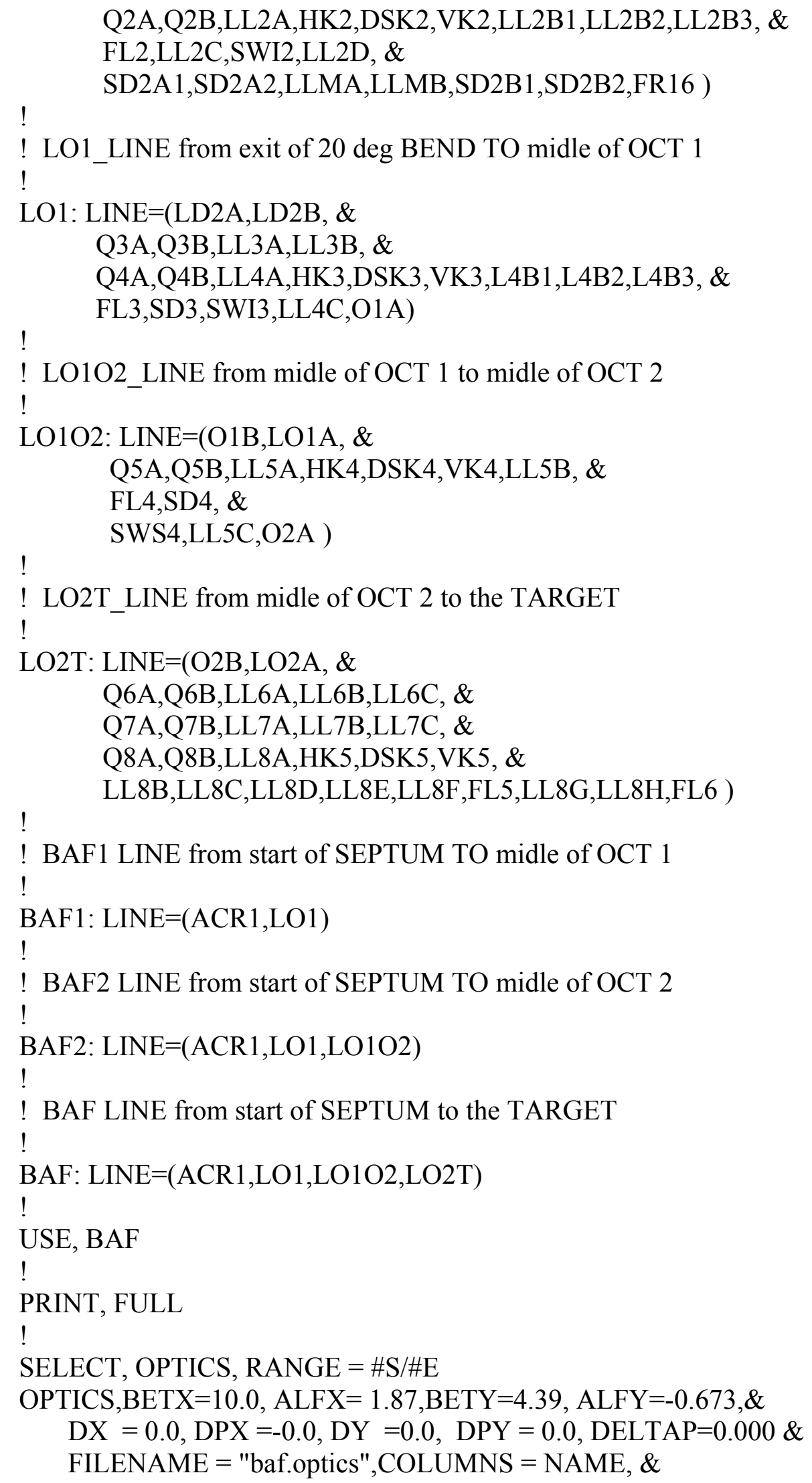


KEYWORD, S, L, K1L, BETX, DX, X, MUX \& , BETY, DY, Y, MUY

!

PRINT, FULL

TWISS, BETX $=10.0, \mathrm{ALFX}=1.87, \mathrm{BETY}=4.39, \mathrm{ALFY}=-0.673, \&$

$\mathrm{DX}=0.0, \quad \mathrm{DPX}=0.0, \mathrm{DY}=0.0, \mathrm{DPY}=0.0$, DELTAP $=0.000$

!

STOP

END 